\title{
Timing of chemotherapy-induced neutropenia is a prognostic factor in patients with diffuse large B-cell lymphoma: a retrospective study
}

Juan Qiu

Shandong Cancer Hospital and Institute, Shandong First Medical University, Shandong Academy of Medical Sciences

Baoxuan Zhang

Shandong Cancer Hospital and Institute, Shandong First Medical University, Shandong Academy of Medical Sciences

Bing Bu

Shandong Cancer Hospital and Institute, Shandong First Medical University, Shandong Academy of Medical Sciences

Shu Fang

Shandong Cancer Hospital and Institute, Shandong First Medical University, Shandong Academy of Medical Sciences

Lihua Song ( $\sim$ SLh9999@VIP.163.com)

Shandong Cancer Hospital and Institute, Shandong First Medical University, Shandong Academy of Medical Sciences

\section{Research Article}

Keywords: Diffuse large B-cell lymphoma, timing, chemotherapy-induced neutropenia, R - CHOP, CHOP, Progression-free survival, overall survival

Posted Date: March 13th, 2021

DOI: https://doi.org/10.21203/rs.3.rs-285196/v1

License: (c) (i) This work is licensed under a Creative Commons Attribution 4.0 International License. Read Full License 


\section{Abstract}

\section{Background}

Chemotherapy-induced neutropenia (CIN) has been shown to be associated with improved clinical outcomes in patients with various solid tumors. The aim of this study was to investigate the relationship between the timing and degree of chemo-induced neutropenia (CIN) and short-term efficacy and survival in newly diagnosed patients with diffuse large B-cell lymphoma (DLBCL).

\section{Methods}

A retrospective study was conducted on 236 newly diagnosed DLBCL patients who received at least 6 cycles of RCHOP (like) or CHOP (like) between January 2012 and December 2018. According to the occurrence time of CIN, subjects were divided into CIN-absent group, early-onset CIN group and late-onset CIN group. According to the degree of CIN, they were divided into CIN-absent group, mild (grade 1-2) CIN group, and severe (grade 3-4) CIN group. Short-term efficacy was evaluated after 4 cycles of treatment. The Kaplan-Meier method was used to draw the survival curve, and the Cox proportional hazards model was applied to determine the correlation between the timing and extent of CIN and clinical features, short-term efficacy, progression-free survival (PFS) and overall survival (OS).

\section{Results}

After 4 treatment cycles, the objective response rate (ORR) of the early-onset group was higher than that of in the lateonset group and CIN absent group (95.7\% VS 88.4\% VS 81.0\%). Multivariate analysis, Ann Arbor staging, choice of treatment plan and CIN timing were the independent prognostic factors for OS and PFS. OS and PFS in the early-onset group were longer than those of in the absent group [OS (HR:0.241; 95\% Cl: 0.110-0.530; $P<0.001)$, PFS (HR: 0.313; 95\% Cl: 0.169-0.579; $P<0.001)$ ] and late-onset group [OS (HR: 0.332; 95\% Cl: 0.161- 0.685; $P=0.003)$, PFS (HR: 0.376; 95\% Cl: 0.204-0.693; $P=0.002)]$

\section{Conclusions}

The timing of $\mathrm{CIN}$ is an independent predictor of prognosis in DLBCL patients treated with R-CHOP (like) or CHOP (like) regimens, and patients with early-onset $\mathrm{CIN}$ have longer survival times. The degree of $\mathrm{CIN}$ is not an independent predictor of prognosis in patients with DLBCL.

\section{Background}

DLBCL is the most common subtype of adult non-Hodgkin's lymphoma [1, 2], and its morbidity and mortality rates have been steadily increasing in China[3,4]. The current first-line standard treatment for DLBCL is a combination of rituximab, cyclophosphamide, doxorubicin, vincristine and prednisone (R-CHOP) or the latter 4 without rituximab (CHOP) [5], both of which can cure about $50-60 \%$ of patients. However, for patients who had failed prior treatment or relapsed after remission, outcomes were particularly poor[6]. In the past, when exploring better initial treatment options, other treatment schemes have failed to show a better effect than $\mathrm{CHOP}$ or R-CHOP; therefore, improving the therapeutic effect of CHOP or R-CHOP and improving DLBCL prognosis are areas worth exploring.

DLBCL is a chemotherapy-sensitive cancer, and the curative effect is proportional to the drug dose to a certain extent; therefore, it is worth studying individualised therapeutic doses to improve the curative effect. Through evaluations before and during treatment, an individualised chemotherapy plan can be made by adjusting the dose in time to determine the ideal amount for each patient. In R-CHOP or $\mathrm{CHOP}$ treatment regimens, the common adverse reaction is 
neutropenia[7, 8], a major factor that leads to the reduction of subsequent doses and additional treatment costs[9, 10]. However, the occurrence of CIN seems to represent dose intensity, and may be related to DLBCL prognosis. Michael et al. [11]studied the relationship between CIN severity and prognosis in 965 DLBCL patients after the first cycle of RCHOP treatment, and found that grade 1-2 CIN had the best prognosis (Grade 0 patients had a $67 \%$-year overall survival rate grade 1-2: 78\%, grade 3: 64\%, and grade 4: 57\%.). Similarly, for gastric cancer[12], lung cancer[13], pancreatic cancer[14], breast cancer[15], colorectal cancer[16] and other diseases, it has been shown that the occurrence of $\mathrm{CIN}$ is related to prognosis. Additionally, CIN timing also seems to be associated with survival, although previous research has shown that the dividing line between early and late occurrence differs. At present, studies have shown that the early onset of CIN in gastric cancer[17], lung cancer[18, 19], pancreatic cancer [20] and colon cancer [21] indicates a longer survival time. However, the relationship between CIN timing and DLBCL prognosis is not clear.

The purpose of this study was to examine the relationship between the timing and degree of CIN in newly diagnosed DLBCL patients treated with R-CHOP and the short-term efficacy and prognosis, in order to improve the initial treatment efficacy of DLBCL and achieve a better prognosis.

\section{Methods}

\section{Patients}

Patients diagnosed with DLBCL and admitted to Shandong Cancer Hospital from November 2011 to December 2018 were enrolled in this retrospective study. Approval was granted by the Ethics Committee of Shandong Cancer Hospital, and all clinical records came from the electronic medical record database of Shandong Cancer Hospital.

The study criteria were as follows: 1) confirmation of DLBCL by pathological diagnosis with no prior treatment; 2) at least 6 cycles of CHOP or CDOP (liposome doxorubicin instead of epirubicin) or R-CHOP or R-CDOP regimen after diagnosis and a standard dose initial treatment regimen; 3 ) no bone marrow infiltration. The exclusion criteria were as follows: 1) incomplete bone marrow toxicity records; 2) lack of follow-up; 3) primary diffuse large B-cell lymphoma of the central nervous system or transformed large B-cell lymphoma of the central nervous system; 4) primary treatment in other hospitals; 5) presence of second or multiple cancers. According to the inclusion and exclusion criteria, 236 patients with DLBCL were identified and chosen for this study. The specific selection criteria are shown in Figure 1.

\section{Treatment regimen and dose intensity}

To reduce the deviation caused by the number of treatment cycles, all patients received at least 6 cycles of first-line treatment with CHOP (like) or R-CHOP (like) regimens. The initial chemotherapy doses were cyclophosphamide (750 $\mathrm{mg} / \mathrm{m} 2, \mathrm{Vi}, \mathrm{d} 1)$, doxorubicin (50 mg/m², Vi, d1-2), vincristine $\left(1.4 \mathrm{mg} / \mathrm{m}^{2}\right.$, maximum $\left.2 \mathrm{mg}, \mathrm{Vi}, \mathrm{d} 1\right)$ and prednisone (100 $\mathrm{mg}, \mathrm{d} 1-5)$ with or without rituximab (375 mg Vi, d0). Each cycle was 21 days. Granulocyte colony-stimulating factor (GCSF) was used when there was grade 3-4 neutropenia, but prophylactic treatment without neutropenia was prohibited.

\section{Assessment of neutropenia}

Routine blood tests were performed every 3-5 days from the first day of chemotherapy until the following cycle. In this study, CIN severity was determined by the minimum absolute neutrophil count (ANC) in the peripheral blood tested during this period. According to National Cancer Institute (NCI) Common Terminology Criteria for Adverse Events (CTCAE, version 4.0), CIN severity is defined as follows: Grade 1, ANC 1.5-2.0 x 109 / L; Grade 2, ANC 1.0-1.5 x 109 / L;

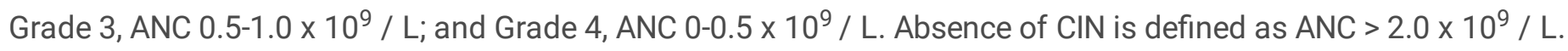
Grade 1 and Grade 2 neutropenia are considered mild neutropenia, and Grade 3 and Grade 4 neutropenia are considered severe neutropenia. According to the CIN occurrence times, CIN occurring during the third cycle of treatment 
was regarded as the bound, early-onset was CIN occurring during weeks 1-3, late-onset was CIN occurring during the fourth cycle or later, and absence of CIN was no CIN occurrence during the entire treatment. Among them, the late-onset group and $\mathrm{CIN}$-absent group were collectively referred to as the non-early-onset group.

\section{Efficacy and survival assessment}

Efficacy was evaluated according to imaging remission (CT/MRI) or metabolic remission (PET/CT)[22] as per the revised 2014 Lugano criteria. Efficacy was evaluated every two cycles after treatment and divided into complete response (CR), partial response (PR), stable disease (SD) and progression disease (PD). The objective response rate (ORR) was the proportion of patients with $\mathrm{CR}$ and PR.

The follow-up end points were OS and PFS, and the follow-up deadline was January 30, 2021. OS is defined as the duration from the beginning of treatment to date of death for any cause. PFS is defined as the duration from the beginning of treatment to time of tumor progression or death of any cause.

\section{Statistical analysis}

IBM SPSS Statistics software (version 22.0) and GraphPad Prism software (version 5.0) were used for the statistical analysis and to generate the graphs. Descriptive statistics was used to describe the baseline characteristics of patients, and a chi-square test was used to compare the baseline characteristics. The Kaplan-Meier method (logarithmic rank test) was used to construct the survival curve. Prognostic factors of OS and PFS were determined through univariate and multivariate Cox regression (enter method) analyses. Variables with $\mathrm{P}<0.1$ from the univariate analysis were entered into multivariate analysis. $\mathrm{P}<0.05$ was defined as a statistically significant difference.

\section{Results}

\section{Demographics}

A total of 236 eligible patients were included in this study. The ratio of males to females was approximately $1: 1.3$, and the median age was 54.9 years (18-80 years). $165 / 236$ patients (69.9\%) were treated with a R-CHOP (like) regimen, and $71 / 236$ patients $(30.1 \%)$ were treated with a CHOP (like) regimen. During treatment, $42 / 236$ patients $(17.8 \%)$ were CINabsent, 166/236 (70.3\%) had early-onset CIN, and 28/236 (11.9\%) had late-onset CIN. In addition to CIN degree, there was a balanced distribution among patients with different CIN timings in terms of sex, age, IPI score, Ann Arbor stage and treatment regimen. Baseline characteristics of the 236 patients and the distribution among groups according to CIN timing are shown in Table 1 (See the table at the end of this article).

\section{CIN and short-term efficacy}

A total of 232 patients who underwent 4 treatment cycles were evaluated by imaging, of which 214 patients achieved ORR. The correlation between the efficacy of 4 treatment cycles and CIN timing is shown in Table 2 (See the table at the end of this article). The ORR of the early-onset group was higher than that of the late-onset group and $\mathrm{CIN}$-absent group (95.7\% VS 88.4\% VS 81.0\%).

\section{Survival analysis}

The median follow-up period of all 236 patients was 42.5 months (5-109 months), and total PFS and total OS were not achieved during the median period. During the follow-up period, 53/236 patients (22.5\%) died and 81/ 236 (34.3\%) relapsed. The Kaplan-Meier survival curves drawn according to CIN timing and CIN degree are shown in Figure 2 and Figure 3, respectively. As shown in Figure 2, when using Kaplan-Meier analysis, every survival curve was meaningful 
regardless of the treatment regimen used, and the OS and PFS of the early-onset group were longer than those of the non-early-onset group.

To evaluate the prognostic values of CIN timing and degree, we performed univariate and multivariate COX regression analysis. The analysis results are shown in Table 3(See the table at the end of this article). Univariate analysis showed that IPI score, Ann Arbor stage, treatment regimen, CIN degree and CIN timing were significant factors affecting the prognosis of OS and PFS. Multivariate analysis showed that Ann Arbor stage, choice of treatment and CIN timing were independent prognostic factors of OS and PFS. The OS and PFS in the early-onset group were longer than those of in the absent group [OS (HR:0.241; 95\% Cl: 0.110-0.530; $P<0.001)$, PFS $(H R: 0.313 ; 95 \% C l: 0.169-0.579 ; P<0.001)$ ] and late-onset group [OS (HR: 0.332; 95\% Cl: 0.161-0.685; $P=0.003)$, PFS (HR: 0.376; 95\% Cl: 0.204-0.693; $P=0.002)$ ]. However, no independent correlation was observed between CIN degree and PFS and OS.

\section{Discussion}

Although $\mathrm{CIN}$ is the most common adverse reaction in cancer patients post-treatment, its occurrence and severity does not represent a poor prognosis. This study is the first to report on the relationship between CIN timing and degree and survival in DLBCL patients. Studies have shown that CIN timing is an independent prognostic factor for patients with DLBCL. We found that the PFS and OS in the early-onset CIN group were significantly longer than those of in the nonearly-onset group (late-onset and absent $\mathrm{CIN}$ ), which is consistent with the findings of previous studies on other types of cancers.

In several different cancers, the early occurrence of CIN represents a good prognosis. He et al.[23] studied the relationship between CIN timing and survival after 6 cycles of carboplatin combined with paclitaxel in patients with primary serous ovarian cancer. They found that the median PFS of the early-onset group VS non-early-onset group was 23 months VS 9 months $(P<0.001)$, and the median OS of the early-onset group VS non-early-onset group was 55 months VS 24 months $(P<0.001)$, and concluded that the early onset of CIN can significantly improve PFS and OS. Similar results have also been found in studies on gastric cancer[17], colorectal cancer [21] and other diseases. Although these findings are consistent with our results, it is worth noting that our results also showed no difference in $\mathrm{PFS} \otimes P=0.645 \rrbracket$ and $\mathrm{OS} \otimes P=0.500 \otimes$ between patients with late-onset $\mathrm{CIN}$ and absent $\mathrm{CIN}$ in the non-early-onset group.

The specific mechanism of the good survival of patients with early-onset CIN is not clear, which may be related to the following points: 1) Early-onset CIN may indicate that patients are sensitive to drugs. Kvinnsland [24]stated that the sensitivity of treatment is a reflection of genetic susceptibility, and the sensitivity of neutrophils and cancer cells to drugs is theoretically the same. Non-early-onset CIN indicates less chemosensitivity and drug resistance, which possibly explains why the short-term effect seen in the early-onset group was better than that of the late-onset group and absent group. 2) CIN reflects the chemotherapy dose and pharmacokinetics of patients. The standard dose of R$\mathrm{CHOP}$ or $\mathrm{CHOP}$ is calculated based on body surface area (BSA). However, due to individual differences in pharmacokinetics and pharmacodynamics, the drug dose calculated by BSA may lead to drug dose deficiency in certain patients[25-27]. CIN occurrence may indicate that a patient received an adequate treatment dose. 3) Neutrophils are related to the progression or prognosis of tumours. Studies have shown that neutrophils play an important role in cancer progression by interacting with cancer cells and immune cells in the blood and tumour microenvironment (TME). They are involved in a variety of functions that promote tumour growth, including proliferation, invasiveness and spread, and immunosuppression[28-30]. In addition, tumour-related neutrophils (TANs) are closely related to a patient's prognosis. Manfroi et al. [31] analysed the relationship between TANs and prognosis in 233 DLBCL patients using the R-CHOP regimen, and used the neutrophil elastase (ELANE) gene as a marker of TAN infiltration. The results 
showed that the high expression of ELANE was associated with decreased overall survival $(H R=2.3,95 \% \mathrm{Cl}$. 1.2-4.3, $P=0.01$ ), which suggests that DLBCL patients with increased TANs may have a poor prognosis.

Additionally, our study shows that CIN occurrence is not an independent prognostic factor of PFS and OS, which differs from the results of Michael et al.[11], who found that grade 1-2 CIN leads to the best prognosis (5-year overall survival rate of patients with grade 0 was $67 \%$, grade $1-2$ was $78 \%$, grade 3 was $64 \%$, and grade 4 was $57 \%$ ). The reasons for the differences in results may be as follows: 1 ) the cut-off points for evaluating CIN degree between the two studies were different. The cut-off point for Michael et al.'s study was the CIN degree after the first treatment cycle, while our study was based on the third cycle. If CIN occurred during the first three cycles, the lowest CIN degree of the previous three cycles was taken as the cut-off point. Otherwise, the cut-off point was the lowest CIN degree that occurred in the following cycle. 2) The total number of treatment cycles was different. There was no requirement for the number of treatment cycles in Michael et al.'s study, but at least 6 treatment cycles were required in this study, which may have excluded certain patients who could not complete 6 cycles of chemotherapy due to age, poor physique, severe myelosuppression and other reasons.

However, there were several limitations in this study. 1) This study was a single-center retrospective study, and the sample size was limited.2) The follow-up time was relatively short, and no end point event occurred in many patients. Despite these limitations, our study still suggests that it seems possible to adjust the dose according to the presence of CIN in 1-3 cycles and choose the appropriate dose for each patient to achieve a better therapeutic efficacy of DLBCL. Of course, this needs to be verified by prospective tests.

\section{Conclusion}

The results of the present study suggest that CIN timing onset is a potential prognostic marker in newly diagnosed DLBCL patients who receive 6 cycles of R-CHOP or CHOP regimens, and early onset CIN leads to a better prognosis.

\section{Abbreviations}

CIN: Chemotherapy-induced neutropenia

DLBCL: Diffuse large B-cell lymphoma

PFS: Progression-free survival

OS: Overall survival

ORR: Objective response rate

G-CSF: Granulocyte colony-stimulating factor

ANC: Absolute neutrophil count

NCl: National Cancer Institute

CTCAE: Common Terminology Criteria for Adverse Events

CR: Complete response

PR: Partial response 
SD: Stable disease

PD: Progression disease

BSA: Body surface area

TME: Tumor microenvironment

TAN: Tumor-related neutrophil

\section{Declarations}

\section{Ethics approval and consent to participate}

The research was performed following international and national regulations in accordance with the Declaration of Helsinki. This study was approved by the Medical Ethics Committee of the Shandong Cancer Hospital (No. SDTHEC2021001023). Prior to treatment, all patients or legal guardians had signed a written informed consent $\square$

\section{Consent for publication}

Not applicable

\section{Availability of data and materials}

The datasets used and/or analysed during the current study are available from the corresponding author on reasonable request.

\section{Competing interests}

The authors declare that they have no competing interests

\section{Funding}

Not applicable.

\section{Authors' contributions}

LHS and JQ carried out the studies, participated in collecting data, and drafted the manuscript. JQ and BXZ performed the statistical analysis and participated in its design. BB and SF helped to draft the manuscript. All authors read and approved the final manuscript.

\section{Acknowledgements}

Not applicable

\section{References}

1. Solans M, Fabrega A, Morea D, Aunon-Sanz C, Granada I, Roncero JM, Blanco A, Kelleher N, Buch J, Saez M et al: Population-based incidence of lymphoid neoplasms: Twenty years of epidemiological data in the Girona province, Spain. Cancer Epidemiol 2019, 58:8-11. 
2. Yoo KH, Lee H, Suh C, Cisl: Lymphoma epidemiology in Korea and the real clinical field including the Consortium for Improving Survival of Lymphoma (CISL) trial. Int J Hematol 2018, 107(4):395-404.

3. Liu W, Liu J, Song Y, Wang X, Zhou M, Wang L, Ma J, Zhu J, Union for China Leukemia Investigators of the Chinese Society of Clinical Oncology UfCLlotCSoCO: Mortality of lymphoma and myeloma in China, 2004-2017: an observational study. J Hematol Oncol2019, 12(1):22.

4. Liu W, Liu J, Song Y, Zeng X, Wang X, Mi L, Cai C, Wang L, Ma J, Zhu J et al: Burden of lymphoma in China, 20062016: an analysis of the Global Burden of Disease Study 2016. J Hematol Oncol 2019, 12(1):115.

5. Tilly H, Gomes da Silva M, Vitolo U, Jack A, Meignan M, Lopez-Guillermo A, Walewski J, Andre M, Johnson PW, Pfreundschuh $\mathrm{M}$ et al: Diffuse large B-cell lymphoma (DLBCL): ESMO Clinical Practice Guidelines for diagnosis, treatment and follow-up. Ann Oncol 2015, 26 Suppl 5:v116-125.

6. Liu Y, Barta SK: Diffuse large B-cell lymphoma: 2019 update on diagnosis, risk stratification, and treatment. Am J Hematol 2019, 94(5):604-616.

7. Bartlett NL, Wilson WH, Jung SH, Hsi ED, Maurer MJ, Pederson LD, Polley MC, Pitcher BN, Cheson BD, Kahl BS et al: Dose-Adjusted EPOCH-R Compared With R-CHOP as Frontline Therapy for Diffuse Large B-Cell Lymphoma: Clinical Outcomes of the Phase III Intergroup Trial Alliance/CALGB 50303. J Clin Oncol 2019, 37(21):1790-1799.

8. Wu J, Song Y, Su L, Xu L, Chen T, Zhao Z, Zhang M, Li W, Hu Y, Zhang X et al: Rituximab plus chemotherapy as firstline treatment in Chinese patients with diffuse large B-cell lymphoma in routine practice: a prospective, multicentre, non-interventional study. BMC Cancer 2016, 16:537.

9. Epstein RS, Aapro MS, Basu Roy UK, Salimi T, Krenitsky J, Leone-Perkins ML, Girman C, Schlusser C, Crawford J: Patient Burden and Real-World Management of Chemotherapy-Induced Myelosuppression: Results from an Online Survey of Patients with Solid Tumors. Adv Ther 2020, 37(8):3606-3618.

10. Li S, Liu J, Bowers C, Garawin T, Kim C, Bensink ME, Chandler DB: Febrile neutropenia-related care and associated costs in elderly patients with breast cancer, lung cancer, or non-Hodgkin lymphoma. Support Care Cancer 2020, 28(1):113-122.

11. Clausen MR, Ulrichsen SP, Larsen TS, Poulsen CB, Tojaga S, Pedersen PT, Madsen J, Pedersen RS, Josefsson PL, Gorlov JS et al: Depth of neutrophil nadir after first cycle of R-CHOP predicts outcome in diffuse large B-cell lymphoma - a nationwide population-based cohort study. Leuk Lymphoma 2019, 60(8):1950-1957.

12. Roviello G, Conca R, D'Angelo A, Multari AG, Paganini G, Chiriaco G, Petrioli R, Corona SP, Rosellini P, Aieta M: Association between neutropenia and response to ramucirumab and paclitaxel in patients with metastatic gastric cancer. Anticancer Drugs 2020, 31(6):632-636.

13. Kishida Y, Kawahara M, Teramukai S, Kubota K, Komuta K, Minato K, Mio T, Fujita Y, Yonei T, Nakano K et al: Chemotherapy-induced neutropenia as a prognostic factor in advanced non-small-cell lung cancer: results from Japan Multinational Trial Organization LC00-03. Br J Cancer 2009, 101(9):1537-1542.

14. Otake A, Tsuji D, Taku K, Kawasaki Y, Yokoi M, Nakamori H, Osada M, Matsumoto M, Inoue K, Hirai K et al: Chemotherapy-induced neutropenia as a prognostic factor in patients with metastatic pancreatic cancer treated with gemcitabine. Eur J Clin Pharmacol 2017, 73(8):1033-1039.

15. Poikonen-Saksela P, Lindman H, Sverrisdottir A, Edlund P, Villman K, Tennvall Nittby L, Cold S, Bechmann T, Stenbygaard L, Ejlertsen B et al: Leukocyte nadir as a predictive factor for efficacy of adjuvant chemotherapy in breast cancer. Results from the prospective trial SBG 2000-1. Acta Oncol 2020, 59(7):825-832.

16. Yoshino T, Cleary JM, Van Cutsem E, Mayer RJ, Ohtsu A, Shinozaki E, Falcone A, Yamazaki K, Nishina T, GarciaCarbonero R et al: Neutropenia and survival outcomes in metastatic colorectal cancer patients treated with trifluridine/tipiracil in the RECOURSE and J003 trials. Ann Oncol2020, 31(1):88-95. 
17. Wang Y, Chen Y, Yin H, Gu X, Shi Y, Dai G: Timing of chemotherapy-induced neutropenia is a prognostic factor in patients with advanced gastric cancer undergoing first-line chemotherapy with oxaliplatin and capecitabine: a retrospective study. Cancer Med 2018, 7(4):997-1005.

18. Jang SH, Kim SY, Kim JH, Park S, Hwang YI, Kim DG, Jung KS: Timing of chemotherapy-induced neutropenia is a prognostic factor in patients with metastatic non-small-cell lung cancer: a retrospective analysis in gemcitabineplus-platinum-treated patients. J Cancer Res Clin Oncol 2013, 139(3):409-417.

19. Lee CY, Park SY, Shin TR, Park YB, Kim CH, Jang SH, Lee JW: Early-onset neutropenia during perioperative chemotherapy is predictive of increased survival in patients with completely resected non-small cell lung cancer: a retrospective analysis. Anticancer Res 2013, 33(6):2755-2761.

20. Chen Y, Shi Y, Yan H, Wang YR, Dai GH: Timing of chemotherapy-induced neutropenia: the prognostic factor in advanced pancreatic cancer patients treated with gemcitabine / gemcitabine-based chemotherapy. Oncotarget 2017, 8(39):66593-66600.

21. Chen Y, Wang Y, Shi Y, Dai G: Timing of chemotherapy-induced neutropenia predicts prognosis in metastatic colon cancer patients: a retrospective study in mFOLFOX6 -treated patients. BMC Cancer 2017, 17(1):242.

22. Cheson BD, Fisher RI, Barrington SF, Cavalli F, Schwartz LH, Zucca E, Lister TA, Alliance AL, Lymphoma G, Eastern Cooperative Oncology $\mathrm{G}$ et al: Recommendations for initial evaluation, staging, and response assessment of Hodgkin and non-Hodgkin lymphoma: the Lugano classification. J Clin Oncol 2014, 32(27):3059-3068.

23. He Y, Li T, Liu J, Ou Q, Zhou J: Early onset neutropenia: a useful predictor of chemosensitivity and favorable prognosis in patients with serous ovarian cancer. BMC Cancer 2020, 20(1):116.

24. Kvinnsland S: The leucocyte nadir, a predictor of chemotherapy efficacy? Br J Cancer 1999, 80(11):1681.

25. Gurney H: How to calculate the dose of chemotherapy. Br J Cancer 2002, 86(8):1297-1302.

26. Cespedes Feliciano EM, Chen WY, Lee V, Albers KB, Prado CM, Alexeeff S, Xiao J, Shachar SS, Caan BJ: Body Composition, Adherence to Anthracycline and Taxane-Based Chemotherapy, and Survival After Nonmetastatic Breast Cancer. JAMA Oncol 2020, 6(2):264-270.

27. Wei CY, Lee MT, Chen YT: Pharmacogenomics of adverse drug reactions: implementing personalized medicine. Hum Mol Genet 2012, 21(R1):R58-65.

28. Demers M, Wong SL, Martinod K, Gallant M, Cabral JE, Wang Y, Wagner DD: Priming of neutrophils toward NETosis promotes tumor growth. Oncoimmunology 2016, 5(5):e1134073.

29. Wculek SK, Malanchi I: Neutrophils support lung colonization of metastasis-initiating breast cancer cells. Nature 2015, 528(7582):413-417.

30. Szczerba BM, Castro-Giner F, Vetter M, Krol I, Gkountela S, Landin J, Scheidmann MC, Donato C, Scherrer R, Singer $\mathrm{J}$ et al: Neutrophils escort circulating tumour cells to enable cell cycle progression. Nature 2019, 566(7745):553557.

31. Manfroi B, Moreaux J, Righini C, Ghiringhelli F, Sturm N, Huard B: Tumor-associated neutrophils correlate with poor prognosis in diffuse large B-cell lymphoma patients. Blood Cancer J 2018, 8(7):66.

\section{Tables}

Table 1 Clinical characteristics of DLBCL patients according to the occurrence time of CIN 


\begin{tabular}{|c|c|c|c|c|c|}
\hline & No.๑\%口 & & Timing of $\mathrm{CI}$ & & $P$ value \\
\hline & $(\mathrm{n}=236)$ & $\begin{array}{l}\text { absent } \quad \text { group } \\
(\mathrm{n}=42)\end{array}$ & $\begin{array}{l}\text { Early-onset } \\
(\mathrm{n}=166)\end{array}$ & $\begin{array}{l}\text { Late-onset } \\
(\mathrm{n}=28)\end{array}$ & \\
\hline Sex & & & & & 0.281 \\
\hline Male & 103(43.6) & $21 \square 50.0 \square$ & $67 \square 40.4 \square$ & $15 \square 53.6 \square$ & \\
\hline Female & $133(56.4)$ & $21(50.0 \square$ & $99 \square 59.6 \square$ & $13(46.4 \square$ & \\
\hline Age (years) & & & & & 0.780 \\
\hline$\leq 60$ & 162(68.6) & $27(64.3)$ & 116(69.9) & 19(67.9) & \\
\hline$\square 60$ & $74(31.4)$ & $15(35.7)$ & $50(30.1)$ & $9(32.1)$ & \\
\hline IPI score & & & & & 0.570 \\
\hline $0-2$ & 146(61.9) & $28(66.6)$ & $104(62.6)$ & $14(50.0)$ & \\
\hline $3-4$ & $83(35.2)$ & $13(31.0)$ & $58(35.0)$ & $12(42.9)$ & \\
\hline $\mathrm{DD}^{1}$ & $7(2.9)$ & $1(2.4)$ & $4(2.4)$ & $2(7.1)$ & \\
\hline $\begin{array}{l}\text { Ann Arbor staging } \\
\text { criteria }\end{array}$ & & & & & 0.338 \\
\hline I-II & $116(49.1)$ & $24(57.1)$ & $81(48.8)$ & 11(39.3) & \\
\hline III-IV & $120(50.9)$ & $18(42.9)$ & $85(51.2)$ & $17(60.7)$ & \\
\hline Treatment regimen & & & & & 0.626 \\
\hline CHOP (like) & $71(30.1)$ & $15(35.7)$ & $47(28.3)$ & $9(32.1)$ & \\
\hline R-CHOP (like] & 165(69.9) & $27(64.3)$ & $119(71.7)$ & $19(67.9)$ & \\
\hline The degree of CIN & & & & & 0.000 \\
\hline Grade 0 & $42(17.8)$ & $42(100.0)$ & $0(0.0)$ & $0(0.0)$ & \\
\hline Grade 1-2 & $94(39.8)$ & $0(0.0)$ & $76(45.8)$ & $18(64.3)$ & \\
\hline Grade 3-4 & $100(42.4)$ & $0(0.0)$ & $90(54.2)$ & $10(35.7)$ & \\
\hline
\end{tabular}

Note: Data are displayed as a count or percentage.

1DDaData Deficient

Table 2 CIN and short-term efficacy

\begin{tabular}{lllll}
\hline & $\mathrm{N}(\mathrm{n}=232)$ & $\mathrm{CR}+\mathrm{PR}(\mathrm{n}=214)$ & $\mathrm{SD}+\mathrm{PD}(\mathrm{n}=18)$ & $P$ value \\
\hline CIN-absent group & 42 & 34 & 8 & 0.01 \\
early-onset group & 164 & 157 & 7 & \\
late-onset group & 26 & 23 & 3 & \\
\hline
\end{tabular}


Table 3 Univariate and multivariate analyses of OS and PFS in relation to clinical parameters 


\begin{tabular}{|c|c|c|c|c|c|c|c|c|}
\hline \multirow{4}{*}{ Variable } & \multicolumn{4}{|c|}{ Overall survival } & \multicolumn{4}{|c|}{ Progression-Free Survival } \\
\hline & \multicolumn{2}{|l|}{ Univariate } & \multicolumn{2}{|l|}{ Multivariate } & \multicolumn{2}{|l|}{ Univariate } & \multicolumn{2}{|l|}{ Multivariate } \\
\hline & $\mathrm{HR} \quad(95 \%$ & $\mathrm{p}-$ & AHR $\quad(95 \%$ & $\mathrm{p}-$ & $(95 \%$ & $\mathrm{p}-$ & AHR $\quad(95 \%$ & $\mathrm{p}-$ \\
\hline & CI) & value & CI) & value & CI) & value & $\mathrm{CI})$ & value \\
\hline \multicolumn{9}{|l|}{ Sex } \\
\hline Male & $0.800(0.466-$ & 0.419 & - & - & $1.012(0.652-$ & 0.958 & - & - \\
\hline Female & $1.373)$ & & & & $1.571)$ & & & \\
\hline \multicolumn{9}{|l|}{ Age } \\
\hline \multicolumn{9}{|l|}{ (years) } \\
\hline$\leq 60$ & $1.408(0.807-$ & 0.229 & - & - & $1.281(0.813-$ & 0.286 & - & - \\
\hline$\square 60$ & $2.457)$ & & & & $2.020)$ & & & \\
\hline \multicolumn{9}{|l|}{ IPI score } \\
\hline $0-2$ & $4.137(2.294-$ & $\square 0.001$ & $2.012(0.983-$ & 0.056 & $3.095(1.959-$ & $\square 0.001$ & $1.406(0.807-$ & 0.229 \\
\hline $3-5$ & 7.461) & & 4.123) & & 4.890) & & $2.449)$ & \\
\hline
\end{tabular}

Ann Arbor

staging

criteria

\begin{tabular}{|c|c|c|c|c|}
\hline I-II & 5.583(2.724- $\square 0.001$ & 4.605(1.906- 0.001 & $4.332(2.563-\square 0.001$ & $4.331(2.243-\square 0.001$ \\
\hline III-IV & 11.441) & 11.126) & 7.321) & 8.363) \\
\hline
\end{tabular}

Treatment

regimen

$\begin{array}{lllllll}\text { CHOP } \square \text { like } \quad 0.368(0.214-\quad \square 0.001 & 0.282(0.156-\quad \square 0.001 & 0.533(0.342- & 0.005 & 0.448(0.278- & 0.001\end{array}$
R-CHOP 0.632)
$0.510)$
0.830)
0.722)

口like[

The degree

of CIN

Grade $\quad 1-2 \quad 0.608(0.309-0.151 \quad 1.061(0.447-0.892 \quad 0.713(0.410-0.232 \quad 1.188(0.586-\quad 0.633$

VS Grade 1.198) 2.521) 2.108)

0

Grade 3-4 0.486(0.241- $0.044 \quad 0.727(0.288-\quad 0.500 \quad 0.488(0.274-\quad 0.015 \quad 0.832(0.379-\quad 0.645$ VS Grade 0.980) 1.836) 1.823)

0

Grade 1- $1.251(0.666-\quad 0.486 \quad 1.460(0.742-\quad 0.273 \quad 1.461(0.881-0.142 \quad 1.428(0.835-\quad 0.193$ 


\begin{tabular}{|c|c|c|c|c|}
\hline $\begin{array}{l}\text { 2VS Grade } \\
3-4\end{array}$ & $2.349)$ & $2.875)$ & $2.422)$ & $2.443)$ \\
\hline \multicolumn{5}{|l|}{ Timing of } \\
\hline \multicolumn{5}{|l|}{ CIN } \\
\hline Early- & $0.410(0.213-0.008$ & $0.241(0.110-\square 0.001$ & $0.481(0.283-0.007$ & $0.313(0.169-\square 0.001$ \\
\hline onset VS & $0.788)$ & $0.530)$ & $0.817)$ & $0.579)$ \\
\hline \multicolumn{5}{|l|}{ Absence } \\
\hline Late-onset & $1.509(0.708-0.287$ & $0.727(0.288-0.500$ & $1.451(0.759-\quad 0.260$ & $0.832(0.379-\quad 0.645$ \\
\hline VS & $3.216)$ & 1.836) & $2.774)$ & 1.823) \\
\hline \multicolumn{5}{|l|}{ Absence } \\
\hline Early- & $0.271(0.139-\square 0.001$ & $0.332(0.161-0.003$ & $0.332(0.189-\square 0.001$ & $0.376(0.204-0.002$ \\
\hline onset VS & $0.531)$ & $0.685)$ & $0.582)$ & 0.693) \\
\hline
\end{tabular}

\section{Figures}


Figure 1

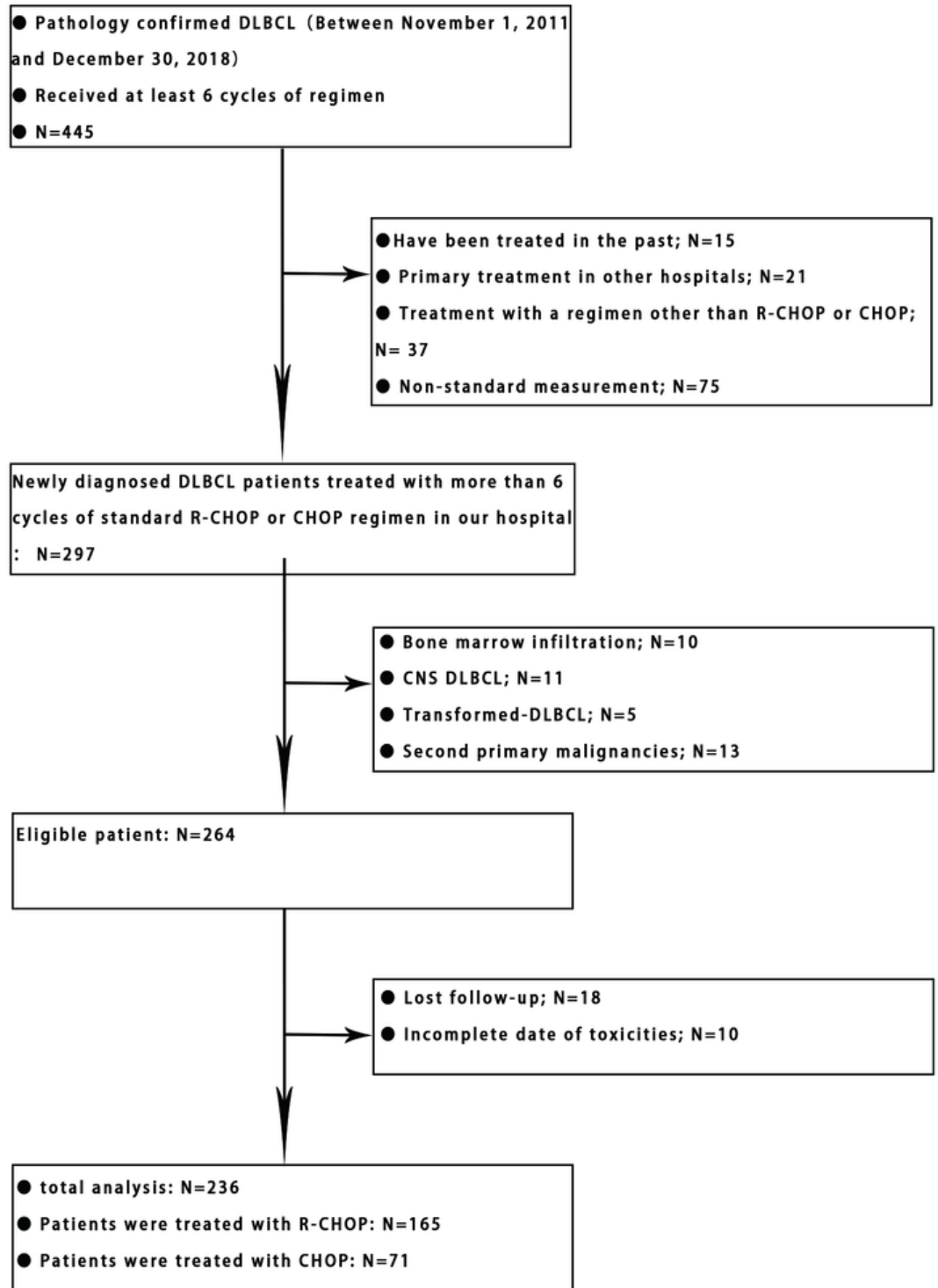

Figure 1

Study flowchart. 


\section{Figure2}

a

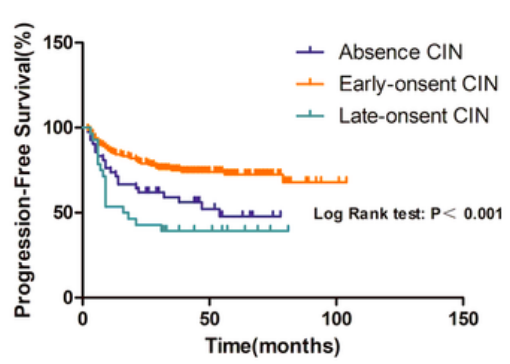

C

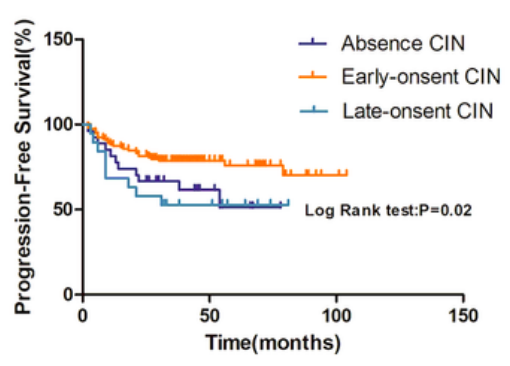

e

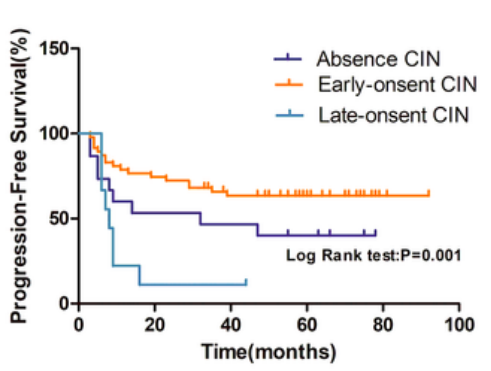

b

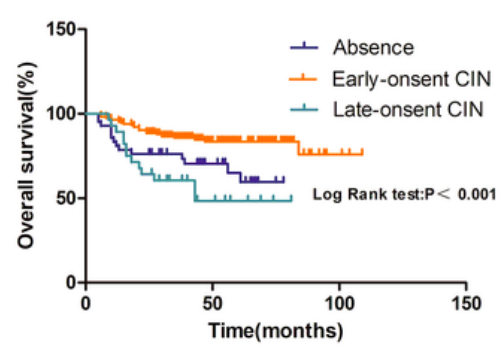

d

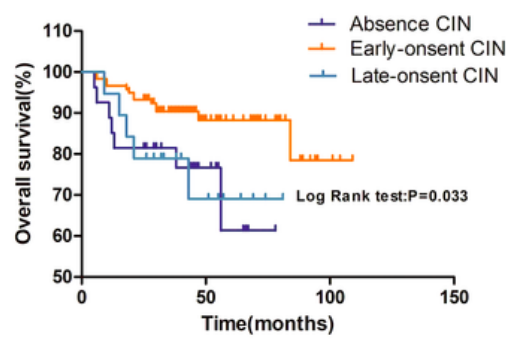

f

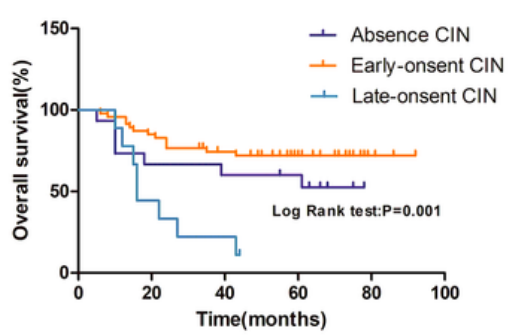

\section{Figure 2}

The Kaplan-Meier survival curves of total PFS (a) and total OS (b) were stratified according to the timing of CIN occurrence, and the Kaplan-Meier survival curves of PFS (c) and OS (d) treated with R-CHOP regimen were used, and the Kaplan-Meier survival curves of PFS (e) and OS (f) treated with CHOP regimen were used. No matter what treatment regimens were used, PFS and OS in the early-onset group were better than those in the non-early-onset group. 


\section{Figure3}

a

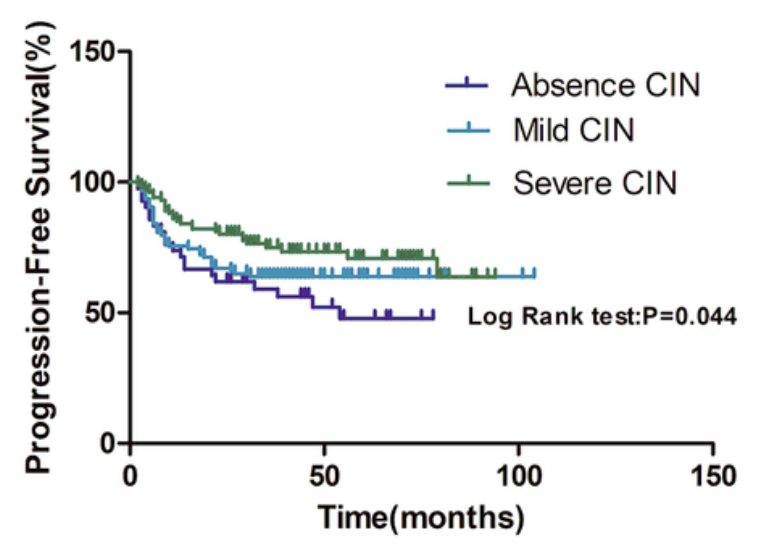

b

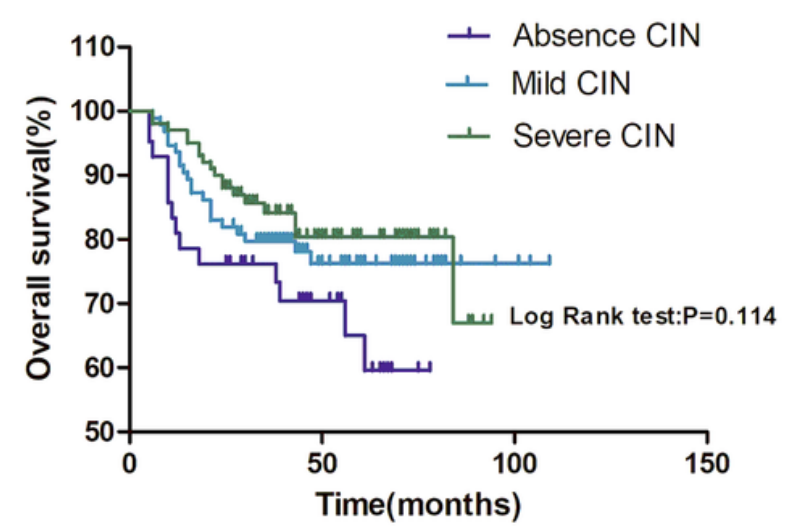

Figure 3

According to the stratification of the occurrence degree of CIN, the survival curves of PFS and OS are shown in figures 3a and 3b. 3a: The PFS of severe (grade 3-4) CIN group was better than that of mild (grade 1-2) CIN group and CIN deletion group. 3b: There was no significant difference in OS among the severe CIN group, mild CIN group and CIN deletion group. 